(Aus dem physiologischen Institut der Universität Marburg.)

\title{
Über den Einfluss der Belastung auf den Kontraktionsakt.
}

II.

Wirkung von Spannungsäudermngen auf die isometrische Summationszuckung.

Von

Dr. John Seemann, Marburg a./L.

(Hierzu Tafel XII und X!II.)

Aus den Untersuchungen Schenck's ${ }^{1}$ ), welche Dehnbarkeit der Muskel in den einzelnen Zeitabschnitten der Tätigkeit besitzt, ergibt sich, dass während summierter Zuckungen die Dehnbarkeit in ihrer Grösse beständig schwankt, und zwar so, dass sie auf den Gipfeln ihre grössten Werte aufweist, nach dem Beginn der neuen (summierten) Zuckung aber jedesmal zu einem Minimum absinkt. In diesen Versuchen kann die "Dehnbarkeit" nicht als einfach zu definierender physikalischer Begriff angesehen werden, sondern die Verhältnisse liegen wegen der Veränderung der Kontraktion durch die Spannung komplizierter; es spielt ein physiologischer Faktor mit.

Das Fazit der früheren Arbeit $^{2}$ ) ist, dass sowohl plötzliche Anspannung des Skelettmuskels als plötzliche Entspannung desselben während der Kontraktion in der gleichen Weise die isometrische Zuckungskurve verändern, indem sie die Spannung bedeutend herabsetzen. Diese Erscheinung konnte im Einklang mit geltenden Anschauungen dahin erklärt werden, dass diese Wirkung zustande kommt nicht durch einen einfachen direkten mechanischen Einfluss

1) F. Schenck, Über die Dehnbarkeit des tätigen Muskels. Sep.-Abdr. aus der Festschrift zum 70. Geburtstag Fi ck's S. 18 ff. 1899.

2) Seemann, Pflüger's Arch. Bd. 106 S. 420. 
auf die kontrahierte Muskelsubstanz, sondern dass es sich um eine physiologische Wirkung auf den Kontraktionsakt handelt: sie beschleunigen beide den Eintritt des zweiten, des kontraktionslösenden Muskelprozesses. Die Frage, wie sich dieser physiologische Faktor in summierten Zuckungen geltendmacht, wurde Veranlassung zu einer Untersuchung darüber, welchen Einfluss plötzliche Spaunungsänderungen im Verlaufe von Summationszuckungen zum Gefolge haben.

Diese Versuche haben aber eine erhöhte Bedeutung und tieferes Interesse aus folgendem Grunde: Bei summierten Zuckungen muss nach Schenck ${ }^{1}$ ) die Annahme gemacht werden, dass die durch den zweiten Reiz ausgelöste neue Kontraktion den Erschlaffungsprozess der ersten Zuckung verzögert. In dem Umstand, dass bei einfachen Zuckungen eine Spannungsänderung bereits im Latenzstadium ihre Wirkung äussert, kann ein Hinweis darauf erblickt werden, dass ein derartiger verzögernder Einfluss auch jedem Einzelreiz schon zukommen wird ${ }^{2}$ ). Fs scheinen also der Reiz (resp. die Erregung) einerseits und die Spannungsänderung anderseits den zweiten Muskelprozess in entgegengesetztem Sinne zu beeinflussen; und dieser Antagonismus beider gibt den aus den einleitend erwähnten Gründen unternommenen Versuchen eine grössere Wichtigkeit.

Die Anordnung der Versuche, welche wieder meistens am Doppelsemimembranosus angestellt wurden, war im grossen und ganzen dieselbe wie früher; nur wurden statt des einen Reizkontaktes unter der Schreibtrommel deren zwei über der Trommel aufgestellt, welche durch zwei auf der Trommel aufgeschraubte Stifte kurz nacheinander geöffnet wurden. Der untere, sonst zur Reizung verwendete Kontakt war dann in den Magnetisierungsstrom eingeschaltet, welcher die Arretierung des Stablhebels bis zum gewünschten Moment besorgte.

Eine Vorfrage war zunächst noch zu entscheiden, nämlich die, ob die plötzliche Dehnung des tätigen Muskels nicht dauernde Veränderungen seiner Substanz hervorruft. Dass das kaum der Fall sein würde, war zu vermuten aus den in der ersten Arbeit mitgeteilten Versuchen, bei denen Dehnung oder Entlastung, kurz vor einer Einzelzuckung vorgenommen, keine oder minimale Veränderungen an der Zuckungskurve erzeugte. Immerhin bandelte

1) Schenck, Pflüger's Arch. Bd. 96 S. 431.

2) Pflüger's Arch. Bd. 106 S. 450. 
es sich dabei um Änderung der Spannung des ruhenden Muskels; der tätige hätte möglicherweise sich anders verhalten können. Versuche zur Entscheidung dieser Frage waren aber leicht anzustellen. Durch passende Verschiebung der die Reizkontakte öffnenden Stifte. wurde das Reizintervall etwas grösser als die Dauer einer Zuckung gemacht, so dass auf die erste isometrische Zuckung sogleich eine zweite folgte. Im ersten Versuch wurden beide Kurven als normale, unbeeinflusste gezeichnet; unmittelbar hinterher wurde dann ein neuer Versuch angestellt, in welchem während der ersten Zuckung der Muskel in der früher beschriebenen Weise plötzlich gedehnt wurde, während die zweite Zuckung nicht gestört wurde. So erhaltene Kurven sind in Fig. 1 abgebildet; die beiden zweiten Kurven fallen ineinander; das zeigt also, dass eine dauernde Beeinflussung oder gar Beeinträchtigung des Muskels durch die angewandten Manipulationen nicht bedingt wurde.

Einige weitere Figuren, Figur 2, 3, 4 und 5, sollen illustrieren, dass die gleichen Tatsachen wie an den einfachen sich auch an summierten Zuckungen konstatieren lassen.

Figur 2 zeigt in der oberen Reihe eine isometrische Summationskurve (1) - die beiden senkrecht stehenden Bogen deuten die Reizmomente an -, ferner die zweite isometrische Zuckung allein ( 7 ) und eine Reihe von Summationskurven, in deren Verlauf eine Dehnung vorgenommen wurde $(2-6)$. Der besseren Übersichtlichkeit wegen ist in der unteren Reihe noch einmal die Kurve 6 der oberen Reihe vom Muskel mit einer isometrischen Summationskurve aufgenommen. Die Spannung, die auf dem Gipfel $850 \mathrm{~g}$ beträgt, sinkt auf $120-150 \mathrm{~g} a b$; an der entsprechenden Stelle hat die ungestörte isometrische Kurve noch $720 \mathrm{~g}$ Spannung.

Die Figur 3 gibt eine ähnliche Serie von Entlastungsversuchen wieder; die Kurven 1 und 2 sind Summationskurven mit geringer oder um $100 \mathrm{~g}$ erhöhter Anfangsspannung, 9 und 8 die entsprechenden zweiten Einzelzuckungen; $3-7$ sind Entlastungszuckungen von dem in der vorigen Abhandlung ${ }^{1}$ ) , besprochenen Typus.

Die Figur 4 soll beweisen, dass auch die plötzlich eintretende, aber andauernde Dehnung ebenso wirkt wie die rasch vorübergehende in Figur 2. Die in derselben übereinandergeschriebenen Kurven haben folgende Bedeutung. Die Kurve 1 ist die isometrische 
Summationskurve ohne wesentliche Anfangsspannung, 2 die Summationskurve mit erhöhter, etwa $80 \mathrm{~g}$ betragender Anfangsspannung, 3 die zweite Einzelzuckung mit erhöhter $(80 \mathrm{~g})$ Anfangsspannung und 4 die Dehnungszuckungskurve, bei der während einer ohne Spannung beginnenden Zuckung der Muskel plötzlich und danernd um den genannten Betrag gedehnt wurde, nach dem Verfahren, welches oben beschrieben wurde. An dem Knie der Kurve, von dem an der Spannungsverlauf wieder richtig, unbeeinflusst von den Schwingungen des Zeichners, wiedergegeben wird, beträgt die Spannung ca. $280 \mathrm{~g}$; zur entsprechenden Zeit weist die zweite Einzelzuckung ca. $630 \mathrm{~g}$, die summierte Zuckung $750 \mathrm{~g}$ Spannung auf.

Die Figur 5 endlich gibt ein paar Versuche an tetanisierten Doppelsemimembranosi; die Kurven zeigen, welche enorme Wirkung durch die relativ geringfügigen Änderungen der Spannung an dem Betrage der durch die Kontraktion entwickelten Spannkraft bewirkt werden können. In dem Versuch $a$ weist nach der Entlastung um ca. $120 \mathrm{~g}$ die isometrische Tetanuskurve an der durch das Kreuz markierten Stelle nicht ganz $300 \mathrm{~g}$ auf an Stelle von $1400 \mathrm{~g}$ der nicht gestörten Kontraktion; in dem zweiten Versuch (b), bei dem die Entlastung in einem früheren Stadium des Tetanus erfolgte, sinkt die Spannung auf $300 \mathrm{~g}$, während die normale Kurve an entsprechender Stelle 1100-1200 g Ordinatenwert hat. Etwas geringer fiel in diesem Versuch die Wirkung der plötzlichen Anspannung aus; die Kurven in der Reihe $c$ illustrieren das Verhalten; in anderen Fällen hatte aber auch der Zug eine ähnlich starke Wirkung wie in dem mitgeteilten Versuchsbeispiel die Entlastung.

Angestellt wurden solche Tetanusversuche ähnlich wie die Summationsversuche, nur wurden die beiden oberen Kontakte in etwas anderer Weise verwendet. Der erste Kontakt wurde als Kurzschlussschlüssel in den sekundären Stromkreis eines Induktionsapparates mit spielendem Wagner'schen Hammer eingeschaltet; der zweite Kontakt befand sich im primären Stromkreis, seine Öffnung hob den primären Strom auf. So konnte der Beginn der einzelnen Tetani in den gleichen Moment verlegt werden; und auch das Aufhören des Tetanus erfolgte in den einzelnen Versuchen nahezu nach gleicher Zeit, welche beliebig kurz bemessen werden konnte.

Diese Ergebnisse gleichen also denen der mit einfachen Zuckungen angestellten Versuche. Auffallend sind die weitaus stärkeren Wirkungen, welche die auf dem Summationsgipfel 
oder im Tetanus vorgenommenen Spannungsäuderungen nach sich ziehen.

Für die ausdrückliche Feststellung sowie zur Erklärung dieser auffallenden Erscheinung, ferner zur Beantwortung der eingangs aufgeworfenen Frage nach dem Antagonismus von Reiz und Spannungsänderung waren zunächst die Wirkungen zu vergleichen, welche eine im gleichen Zeitmoment vorgenommene Spannungsänderung auf die Einzelzuckungen und auf die summierte Zuckung hat. Um nicht zu komplizierte Verhältnisse zu bekommen, liess ich die zweite Zuckung auf der Höhe der ersten einsetzen; bei konstant beibehaltenem Intervall der Reizmomente war dann während der Summationszuckung und während jeder der beiden Einzelzuckungen der Muskel im gleichen Zeitpunkte zu dehnen resp. zu entləsten.

Solche Versuche ergaben bemerkenswerte, regelmässig eintretende Unterschiede in Grösse der Wirkung je nach der Lage des Zeitpunktes, in welchem die Spannungsänderung erfolgte, zu dem zweiten Reizmoment. An der Hand der in den Figuren 6-10 wiedergegebenen Kurven solcher Versuchsserien will ich zunächst die Ergebnisse zusammenfassen und die Deutung und Analyse der Befunde dieser Beschreibung folgen lassen.

Die abgebildeten Versuche betreffen meistens solche mit Dehnung; ich habe diese zur Illustration gewählt, weil die an sich schon schwer zu übersehenden Kurvenbilder in den Entlastungsversuchen noch verworrener erscheinen. Eine Serie von Entlastungsversuchen gibt die Figur 10 wieder, in welcher mit 1 die Summationszuckung, mit 2 die zweite und mit 3 die erste Einzelzuckung, wie sie unter dem Einfluss der Entspannung um etwa $100 \mathrm{~g}$ sich ergaben, bezeichnet wurden. Die senkrechten Bogenlinien bezeichnen den zweiten Reizmoment. Die Resultate sind in dem Versuch von Fig. 10 wie in ähnlichen Versuchen die gleichen wie bei den sofort zu behandelnden Dehnungsversuchen.

Setzt die Dehnung vor dem zweiten Reizmoment ein, so ist zu erwarten, dass ein Abfall der Spannung eintritt entsprechend dem Abfall der ersten Einzelzuckung; zu dem noch verbleibenden Rest von kontraktiler Kraft muss sich der Effekt der zweiten Reizung summieren; derselbe kann an sich ja nicht von der Dehnung beeinflusst sein, weil sie vor seinem Eintritt beendet ist. Das geschieht auch (cf. Fig. 2 Kurve 3, Fig. $7 e$ und $9 a$ ), und zwar in derselben Weise wie bei der Summation überhaupt, wenn die summierte Zuckung auf 
dem Gipfel der ersten einsetzt; die so veränderte Summationskurve verläuft über und annähernd parallel mit der zweiten Einzelzuckung.

Wenn in einem etwas später gelegenen Zeitpunkt der Kontraktion am Muskel gezogen wird, so dass die Dehnung oder ihre Nachwirkung sich noch in die zweite Zuckung hinein erstreckt (Fig. 2, Kurve 4) oder dass sie überhaupt erst im Anfang der zweiten Zuckung erfolgt (Fig. 6b), so muss sie auch auf diese wirken; und wenn sie das tut, muss die Höhe der Dehnungszuckungskurven relativ rasch sinken (Fig. 2, Kurve 4 und 5 ; Fig. $9 a$, Zuckung 2 und 3 ; Vergleich zwischen $6 a, 7 b$ und $8 a$ ). Immerhin bleibt aber in diesen Fällen die Summationszuckung mit Dehnung über derjenigen, welche die Einzelzuckung nach einer zu gleicher Zeit erfolgenden Dehnung liefern würde; die Spannung bleibt grösser oder mindestens gleich der Summe der Spannungen, welche die an gleicher Stelle gedehnten Einzelzuckungskurven aufweisen.

Das wird aber anders, wenn man sich mit dem Moment der Dehnung dem "Gipfel der summierten Zuckung nähert; schon das Beispiel $8 a$ ist ein Uebergang $\mathrm{zu}$ der Erscheinung, welche bei Dehnung auf dem Gipfel eintritt. Dann liegt der weitere Verlauf der summierten Zuckung in oder meistens sogar unter der Bahn der Kurve der gedehnten zweiten Einzelzuckung (Fig. 8b, 9b,6a). Eine Wiederholung solcher Umstände kann natürlich eine weitere Steigerung noch hervorrufen, und das kann hinreichen, um die starke Spannungsherabsetzung im Tetanus verständlich zu machen.

Die Erklärung dieser Befunde ist leicht zu geben auf Grund der früher entwickelten Vorstellungen, welche sich an die PflügerFick'sche Kontralktionshypothese anschliessen.

Wenn die Dehnung vor dem zweiten Reizmoment vorgenommen wird, so bietet die Wirkung nichts Besonderes; es erfolgt eine Kontraktion, wie oben angedeutet, ganz nach den Regeln der Summation. Erfolgt die Dehnung aber im Bereich der zweiten Zuckung, so werden die Verhältnisse komplizierter.

Auf dem Gipfel einer Zuckung hat der Erschlaffungsvorgang wieder mindestens die gleiche Intensität wie der gleichzeitig sich noch abspielende Teil des eigentlichen Kontraktionsprozesses. Durch den neuen Reiz soll die Erschlaffung verzögert werden, und da sich von der zweiten Zuckung zunächst natürlich der erste Prozess überwiegend entwickelt, so muss bei einer summierten Zuckung die Interferenz der beiden Prozesse von neuem stark zugunsten des 
ersten gestört sein ${ }^{1}$ ); es muss aber durch die Verzögerung ein grosser Teil des Erschlaffungsprozesses aufgespart, gewissermassen im Vorrat gehalten sein. Erfolgt nun eine Dehnung in der summierten Zuckung, so kann sie ihre Wirkung auf einen grösseren Betrag des Erschlaffungsprozesses entfalten. Der Ordinatenwert der Kurve einer solchen Summationszuckung mit Dehnung wird nun so lange die Summe der Ordinaten entsprechend veränderter Einzelzuckungen an Grösse übertreffen, als noch ein solcher Teil der Elementarprozesse sich in oder vor dem ersten Stadium befindet, dass sie über die bis dahin in die zweite Phase eingetretenen Elementarprozesse in der Übermacht sind, und dass auch deren stossweise Beschleunigung durch die Dehnung sie nicht aufkommen lässt gegenüber der Intensität des ersten Prozesses. Das wird im Anfang der zweiten Zuckung der Fall sein, etwa bis zum Wendepunkt dieses zweiten Anstiegs der Summationskurve; von dort ab wird die Beschleunigung des ersten Prozesses negativ. Die Fälle, in denen diẻ Dehnung erst gegen den Gipfel der summierten Zuckung eintritt, lassen also ein derartiges Übergewicht des ersten Prozesses nicht mehr zustande kommen; vielmehr müssen dann die Verhältnisse etwa folgendermassen liegen: Da in den hier mitgeteilten Beispielen die Muskeln eigentlich durchweg etwas ermüdet waren (siehe erste Arbeit, Versuchsanordnung), ist die Gipfelzeit ${ }^{2}$ ) der summierten Zuckung etwas kürzer als die der zweiten Finzelzuckung; es ist also in der summierten Zuckung der ihr zugrunde liegende Prozess überhaupt schon etwas weiter entwickelt als in der Einzelzuckung; dazu kommt nun vor allen Dingen, dass die erste Phase des Kontraktionsprozesses um die Zeit des Gipfels zum grössten Teil bereits durchlaufen ist; der Erschlaffungsvorgang muss mindestens die gleiche Intensität haben wie bei der Einzelzuckung, vermehrt um den von der ersten Zuckung her retardierten Teil. Bei der plötzlichen Auslösung und Beschleunigung des zweiten Prozesses muss man also dann eine stärkere Wirkung erwarten, als wenn nur die in der Einzelzuckung allein vorhandene Erschlaffungstendenz gesteigert wird. Daher muss, wenn die Dehnung um den Gipfel der Summationszuckung geschieht, der Endverlauf der Doppelzuckung unter dem der Einzelzuckung gelegen sein.

1) Cf. Schenck, Pflüger's Arch. Bd. 96, l. c.

2) Cf. hierzu Schenck, Pflüger's Arch. Bd. 96 S. 412. 
Wegen der Verzögerung der noch der ersten Zuckung angehörigen Erschlaffung durch den zweiten Reiz und ihrer beschleunigten Auslösung durch die Dehnung kann aber unter Umständen auch schon vor der Gipfelzeit leicht ein Uberwiegen der Erschlaffung über den positiven Kontraktionsprozess sich zeigen. Daher kommt es; dass sich an Doppelzuckungen relativ häufiger als an Einzelzuckungen die Erscheinung beobachten liess, dass nach dem ersten plötzlichen Absinken die Kurve auch dauernd, natürlich weniger steil als die normale fällt, und zwar vor ihrer eigentlichen Gipfelzeit. Ein Beispiel der Art ist die Kurve 2 der Fig. $9 a$.

Diese Betrachtungen haben also gezeigt, dass man mit Hilfe der Deutung: der Reiz verzögert, die Dehnung beschleunigt den Erschlaffungsvorgang, imstande ist, die aufgefundenen Tatsachen zu erklären. Beide Deutungen sind ursprünglich einzeln aus entsprechenden Versuchen abgeleitet; es erscheint daher der Nachweis wichtig, dass sie auch die Versuchsresultate erklären, welche erhalten werden, wenn Reiz und Dehnung kombiniert oder kurz nacheinander auf den tätigen Muskel einwirken. Was der Reiz von dem Erschlaffungsprozess verzögert hat, kann durch plötzlichen Zug (oder. Entlastung) in beschleunigtem Zeitmass ausgelöst und zur Entwicklung gebracht werden.

Auf diese Versuche und die ibnen gegebene Erklärung lässt sich ein interessantes Experimentum crucis anstellen, dessen Grundlage folgende Überlegung bildet.

Ist die gegebene Deutung richtig, so muss eine Laständerung, welche eine bestimmte konstante Zeit nach dem zweiten Reizmoment erfolgt, in der Regel verschieden stark wirken, wenn bei absteigender Summation das Intervall zwischen den beiden Reizmomenten variiert wird. Je enger das Reizintervall gewählt wird, je näher dem Gipfel also die zweite Zuckung einsetzt, desto weniger wird die der ersten Zuckung zugehörige Erschlaffung sich abgespielt haben, desto grösser ist also der retardierte Teil, welcher durch Spannungssteigerung oder -verminderung beschleunigt werden kann. Es müsste also, wenn die entwickelten Anschauungen Gültigkeit haben, bei engem Reizintervall (Gipfelsummation) die Zugzuckungskurve niedriger sein als bei weitem Reizintervall (Summation im absteigenden Schenkel), und zwischen den beiden Extremen müssen sich kontinuierliche Übergänge finden. Dieser Erfolg ist als Regel zu erwarten, wenn die Dehnung oder Entlastung so zeitig erfolgt, 
dass noch ein ausreichender Teil der ersten Kontraktionsphase vorhanden ist und sich geltend machen kann, also wenn die Dehnung oder Entspannung noch vor dem Gipfel der Summationszuckung vorgenommen wird. Wird erst auf dem Gipfel oder später die Spannung plötzlich geändert, so darf man von der Grösse des Reizintervalles keinen merkbaren Einfluss erwarten, weil dann zur Zeit der Dehnung die Intensität des Erschlaffungsprozesses in jedem Falle (bei weitem wie bei engem Reizintervall) das Maximum erreicht oder gar überschritten hat.

Derartige Versuche habe ich vielfach mit glejchem Ergebnis angestellt. Ich gebe aus meinem Kurvenmaterial nur je eine Serie von Versuchen mit Anspannung und Entspannung (Fig. 11 u. 12). Dieselben zeigen deutlich, dass die erwarteten Phänomene tatsächlich eintreten.

Die Fig. $11 b$ bringt in den beiden ersten Reihen je eine Summationszuckung, die zweite Einzelzuckung und die Zugzuckung der Doppelzuckung, in der ersten Reihe bei engem Reizintervall, in der nächsten bei weiterem Reizintervall. Die dritte Reihe enthält zwei Doppelzuckungen mit verschiedenem Reizintervall und die zweite Einzelzuckung, jede mit der entsprechenden Zugzuckungskurve, bei der die Dehnung kurz nach dem Gipfel geschah.

In den ersten vier Reihen der Fig. 12 sind die Kurven abgebildet, welche durch Entlastung bald nach dem zweiten Reiz bei nach und nach vergrössertem Reizintervall erhalten wurden; die fünfte Reihe zeigt den Einfluss der auf dem Gipfel vorgenommenen Entlastung bei weitem und bei engem Intervall.

Die Versuche entsprechen in ihren Resultaten also vollkommen den gehegten Vermutungen; sie bestätigen übrigens damit den in der vorigen Arbeit und früher von Schenck abgeleiteten Satz, dass die Wirkung der Dehnung (resp. der Entlastung) sich erstreckt auf den zweiten Muskelprozess, denn dieser ist das eigentlich und wesentlich Variierende in den einzelnen Versuchen der Serie.

Durch die geschilderten Versuche ist demonstriert, dass der Reiz und die Spannungsänderung entgegengesetzt auf die zweite Phase des Kontraktionsprozesses wirken. Dieser Antagonismus zwischen Reiz und Spannung scheint aber nicht auf den zweiten Muskelprozess beschränkt zu' sein; auch auf den ersten Prozess baben beide Eingriffe entgegengesetzten Einfluss: Der Einfluss der 
Spannung auf den Kontraktionsvorgang (Heidenhain, Fick) lässt sich dahin zusammenfassen, dass Spannungsvermehrung die Erregbarkeit steigert [Schenck ${ }^{1}$ )]. Durch die Tätigkeit des Muskels wird nach den Versuchen von Schenck und Bradt ${ }^{2}$ ) über die Wärmeentwicklung während summierter Zuckungen die Erregbarkeit herabgesetzt.

Für die Spannungsvermehrung besteht also ein vollkommener Antagonismus von Reiz und Belastung auf den ganzen Kontraktionsakt; für die Entlastung muss eine solche Betrachtung ausgeschlossen bleiben, weil unsere Kenntnisse von ihrer Wirkung nach dieser Richtung hin zwar bislang zu gering sind; weil aber ihre Wirkung anf den ersten Prozess nach allen Vorstellungen, die man über die Wirkung der Spannung hat, nicht mit der Spannungswirkung gleich sein kann. Das zeigt also, dass der Einfluss, den sowohl Spannung als Reiz auf den ersten Prozess ausüben, dem Wesen nach verschieden sein muss von ihrer Einwirkung auf den zweiten. Für das Zustandekommen der Wirkung auf die erste Phase ist Spannungsvermehrung allein erfolgreich und längere Dauer der Belastung von Vorteil, für die auf die zweite Phase dagegen plötzliches Fintreten der Lastveränderungen; auf die Dauer und die Grösse kommt es dabei weniger an, und in dieser Phase sind Entlastung und Anspannung gleich wirksam.

1) Schenck, Pflüger's Arch. Bd. 51 S. 528.

2) Schenek und Bradt, Pflüger's Arch. Bd. 55 S. 143. Cf. auch Hofmann, Pflüger's Arch. Bd. 103 S. 298. 
